\title{
Concomitant plasmacytoma and B cell lymphoma with discordant light chain expression but clonal identity
}

\author{
Wing Y. Au • Lau Wai-Hung • Kai Y. Wong • \\ William W. L. Choi
}

Received: 12 August 2011 / Accepted: 26 February 2012 / Published online: 8 March 2012

(C) The Author(s) 2012. This article is published with open access at Springerlink.com

\section{Dear Editor,}

A 63-year-old patient presented with abdominal pain. A computerized tomogram scan showed a gallbladder mass (Fig. 1a). A positron emission tomogram revealed additional FDG-avid lesions in the sternum, ribs, bilateral humeri, vertebral column, and pelvic bone (Fig. 1b). Magnetic resonance imaging confirmed vertebral lytic lesions (Fig. 1c). A serum protein electrophoresis showed a monoclonal band $(\operatorname{IgA} \lambda 16.2 \mathrm{~g} / \mathrm{l})$, and he was referred for suspected plasma cell myeloma (PCM). A bone marrow biopsy, however, showed $19 \%$ infiltration by monoclonal B cells, expressing CD20, but negative for CD3-, CD5, CD10, CD138, IgA, IgG, and IgM (Fig. 1d). These cells showed $\mathrm{k}$-light chain restriction (Fig. 1e, f). Staining with CD138 highlighted less than $1 \%$ plasma cells, while the lymphoma cells do not express CD138. In view of the discrepancy, cholecystectomy was performed showing a plasmacytoma (Fig. 1g). The plasma cells were CD138 positive, CD20 negative, and also positive for cytoplasmic lambda chain and IgA, but negative for IgG, IgM, and $\kappa$-light chain restriction. (Fig. 1h, i). They also expressed CD56 and cyclin D1. The double

\footnotetext{
W. Y. Au ( $\square)$

Department of Medicine, Queen Mary Hospital, The University of Hong Kong,

UMU, 4/F, Professorial Block Pokfulam Road,

Pok Fu Lam, Hong Kong

e-mail: auwing@hotmail.com

L. Wai-Hung $\cdot$ K. Y. Wong $\cdot$ W. W. L. Choi

Department of Pathology, Queen Mary Hospital,

The University of Hong Kong,

Pok Fu Lam, Hong Kong
}

pathology was treated with R-CEOP $\times 6$ (rituximab, cyclophosphamide, epirubicin, vincristine, and prednisolone) together with maintenance with thalidomide and bortezomib (weekly $\times 16$ doses) and radiotherapy to both humerus. A complete remission by radiological and serum immunofixation assessment was shown at 1 year. $\mathrm{He}$ refused an autologous stem cell transplantation (SCT). The clonal relationship between the two lesions was investigated by polymerase chain reaction using IgH VJ primers followed by cloning and sequencing, using DNA from the homogeneous plasmacytoma and microdissected lymphoma cells from the trephine biopsy [1]. This showed a direct relationship between the two B cell clones in terms of band size and sequence homology (Fig. 1j and k).

This case is interesting in terms of pathogenesis and treatment. In terms of pathogenesis, cases of concomitant B cell lymphoproliferation and plasma cell dyscrasia are rare and usually of independent clones [2]. The immunophenotyping and light chain expression do not suggest a common evolution from a lymphoplasmacytoid lymphoma. Our case is characterized by clonal homology but discordant light chain expression, immunophenotype, and morphology. Plasma cell myeloma with discordant light chain secretion is well documented [3]. Posttreatment switch in monoclonal protein may also occur after SCT [4]. More dramatically, changes in immunophenotype, morphology, and light chain expression in B cell malignancies after rituximab treatment are also recognized $[1,5,6]$. However, in our case, the clones occurred concomitantly and upfront in distinct cell lineages. This may theoretically result from plasma cell differentiation of the malignant $\mathrm{B}$ lymphocytes or a divergent evolution from a primordial B cell clone. Pools of malignant clonotypic B cells are known to exist in PCM patients [7]. In terms of treatment, 


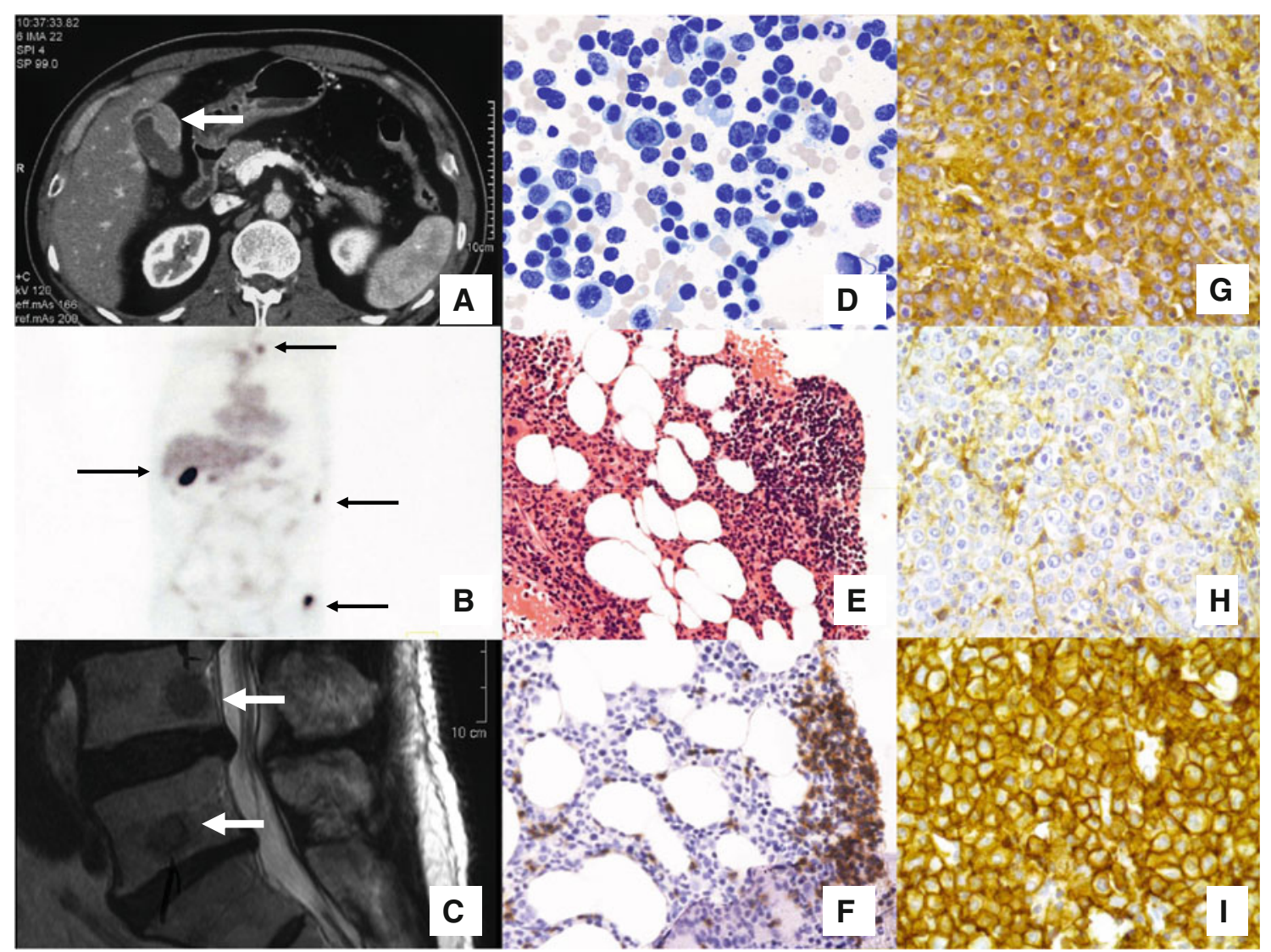

$\mathbf{J}$

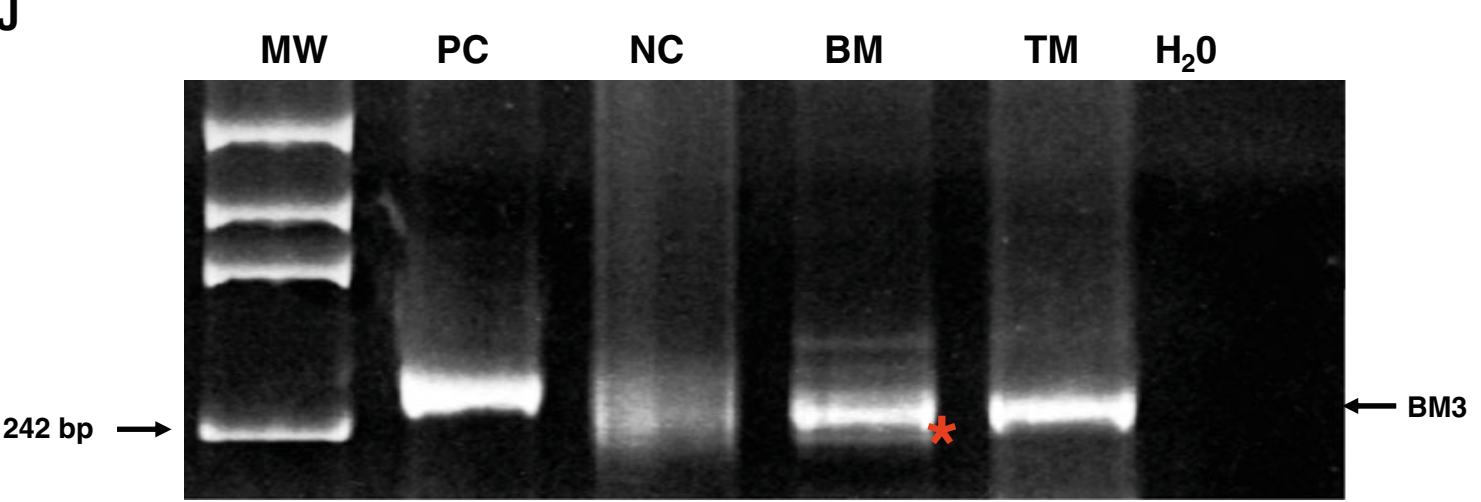

K

TM GTCCTGCAGGCCTCCGGGAAGGGTCTGGAGTGGATTTCATACATTAGTGGTAATAGTCGT 60 BM3 GTCCTGCAGGCCTCCGGGAAGGTCTGGAGTGGATTTCATACATTAGTGGTAATAGTCGT 60 GTCCTGCAGGCCTCCGGGAAGGGTCTGGAGTGGATTTCATACATTAGTGGTAATAGTCGT

TM

ACCATGTACTACGCAGACTCTATCAAGGGCGATTCACCATCTCCAGAGACGACGCCAAA 120 BM3 ACCATGTACTACGCAGACTCTATCAAGGGCCGATTCACCATCTCCAGAGACGACGCCAAA

TM AACTCAGTGTTTCTGCAAATGAACAGCCTGAGCGCCGAAGACACGGCTATTTATTATTGT 180 BM3 AACTCAGTGCTTCTGCAAATGAACAGCCTGAGCGCCGAAGACACGGCTATTTATTATTGT 180

TM

GCGAGAGGGGGGATAATTTTTATACTACCTTGGACTCCTGGGCCAAGGGATCCTGGTCA 240 BM3 GCGAGAGGGGGGAATAATTTTTATACTACCTTGGACTCCTGGGCCAAGGGATCCTGGTCA

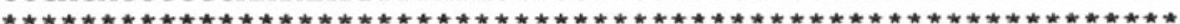

TM CCGTCTCCTCA 251

BM3 CСGTCTCCTCA 251

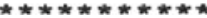


4 Fig. 1 a Computerized tomogram scan showing a gallbladder lesion (arrow). b Positron emission tomogram scan showing FDG avid uptake (arrows) in the porta hepatis as well as in the sternum, ribs, and femur. c Magnetic resonance imaging showing osteolytic lesions in multiple vertebra levels. d-f Marrow aspirate and trephine biopsy showing heavy lymphoma infiltration with no evidence of plasma cell dyscrasia. The B cells were highlighted by CD20 staining (f). g-i The gallbladder lesion showed sheets of plasma cells with CD138 expression (g) negative for kappa light chain (h) but expressing lambda light chain (i). j Polymerase chain reaction using IgH primers on DNA extracted from marrow and gallbladder lesions showing bands of similar band size (asterisk and arrow). $M W$ molecular weight control, $P C$ positive control, $N C$ negative control, $B M$ bone marrow, $T M$ tissue material from gallbladder, $\mathrm{H}_{2} \mathrm{O}$ water blank. k Cloning, sequencing, and alignment showing homology between clones from the two discordant lesions

a combination of lymphoma and PCM treatment seems to be effective in controlling both lesions with minimal overlap of toxicity. It is uncertain whether this patient may have survival benefit from an autologous SCT.

Open Access This article is distributed under the terms of the Creative Commons Attribution License which permits any use, distribution, and reproduction in any medium, provided the original author(s) and the source are credited.

\section{References}

1. Au WY, Srivastava G, Wong KY, Chung LP, Ma SK, Wan TS et al (2004) Transformation of diffuse large B-cell lymphoma into pre-B acute lymphoblastic leukemia: clinicopathologic features and clonal relationship. Hum Pathol 35(7):900-903

2. Pantic M, Schroettner P, Pfeifer D, Rawluk J, Denz U, SchmittGraff A et al (2010) Biclonal origin prevails in concomitant chronic lymphocytic leukemia and multiple myeloma. Leukemia 24(4):885890

3. Ando K, Yaguchi M, Okabe S, Miyazawa K, Ohyashiki K (2000) IgA-lambda/IgG-kappa biclonal myeloma in which two clones proliferated in individual sites. Intern Med 39(2):170175

4. Zent CS, Wilson CS, Tricot G, Jagannath S, Siegel D, Desikan KR et al (1998) Oligoclonal protein bands and Ig isotype switching in multiple myeloma treated with high-dose therapy and hematopoietic cell transplantation. Blood 91(9):3518-3523

5. Hwang YY, Wong KY, Leung RY, Wong SH, Chan SC, Srivastava G et al (2009) Post-rituximab Burkitt transformation of PTLD: loss of CD20 expression accompanied by a switch in light-chain expression. Ann Hematol 9:9

6. Au WY, Wong K, So JC, Wan TS, Srivastava G, Liang R (2009) A unique case of B cell lymphoma relapsing as CD4/CD56 blastic neoplasm. Leuk Lymphoma 50:932-936

7. Conway EJ, Wen J, Feng Y, Mo A, Huang WT, Keever-Taylor CA et al (2009) Phenotyping studies of clonotypic B lymphocytes from patients with multiple myeloma by flow cytometry. Arch Pathol Lab Med 133(10):1594-1599 